\title{
Quelles synergies entre connaissances scientifiques et empiriques? L'exemple des cultures du safran et de la truffe
}

\author{
Nathalie Girard ${ }^{\mathrm{a}}$, Mireille Navarrete ${ }^{\mathrm{b}}$ \\ a Ingénierie des connaissances, INRA, SAD Sicomor, BP 27, 31326 Auzeville cedex, France \\ b Agronomie, INRA SAD Écodéveloppement, Domaine Saint-Paul, Site Agroparc, 84914 Avignon cedex 9, France
}

\begin{abstract}
Pour poursuivre les réflexions sur la prise en compte des connaissances et des savoir-faire des praticiens dans le raisonnement scientifique, ici celui des agronomes, cet article ébauche les bases d'une approche transdisciplinaire. Elle ne vise pas seulement à intégrer des connaissances empiriques dans la démarche scientifique, mais à caractériser une diversité d'approches croisées. Le cas des deux cultures présentées ici, la truffe et le safran, en fournit un support particulièrement pertinent, car, exploitées par des producteurs d'une grande diversité, pas forcément agriculteurs, elles sont très mal connues des agronomes. De surcroît, elles se cachent sous la terre! Au lecteur d'en découvrir les mystères. .
\end{abstract}

La Rédaction

\section{Mots-clés :}

safran;

truffe ;

agronomie ; connaissances empiriques ; connaissances scientifiques ; ingénierie

\section{Keywords:}

saffron;

truffle;

agronomy; empirical knowledge; scientific knowledge; engineering
Résumé - Dans un contexte de redéfinition des fonctions de l'agriculture dans la société, les «systèmes de connaissance agricole » doivent concevoir de nouveaux modes de collaboration entre agriculteurs, agents de développement et scientifiques, mais aussi renouveler les connaissances à produire. Peu de travaux portent sur le contenu des connaissances à construire dans ces situations. Ce texte part de l'hypothèse que des connaissances agronomiques peuvent émerger d'une synergie entre connaissances empiriques des praticiens et connaissances scientifiques. Il s'attache à explorer cette nouvelle forme de production de connaissances, à partir des cas du safran et de la truffe, deux cultures dont la maîtrise technique constitue un frein majeur à leur développement. En confrontant les données sur les connaissances des safraniers et trufficulteurs à la littérature scientifique et technique existant sur ces cultures et à la théorie agronomique, ce texte propose quatre modes de combinaison entre connaissances empiriques et scientifiques.

\begin{abstract}
Exploring synergies between scientific and empirical knowledge: the case of saffron and truffle cropping in France. Knowledge systems in agriculture need to devise new forms of collaboration between farmers, development agents and researchers in the current socio-economic context in which the social functions of agriculture are being questioned. So far very few research studies have addressed the issue of the knowledge content to be produced in such situations. Our paper rests on the hypothesis that the synergy between the empirical knowledge of producers and scientific knowledge is able to generate new agronomic knowledge. We explored this new form of knowledge production by examining two situations: saffron cultivation in southwestern France and French truffle production, for which the technical management is a major hindrance to their development. We show that neither scientific nor empirical knowledge on their own are sufficient to improve it. On the basis of an "engineering of agronomic knowledge" approach that makes explicit and combines knowledges from different sources, we then propose four modes of synergy: (1) translating the objectives and practices of producers into action-oriented scientific questions; (2) scientifically grounding heuristic knowledge by referring it to explicative scientific knowledge; (3) linking the indicators used by the producers to scientifically measurable parameters; (4) selecting on a scientific basis appropriate equivalent plants for transposing knowledge from one to the other. These four modes are then discussed; it is underlined that this cognitive approach to the synergy between empirical and scientific knowledge could effectively be completed by analysing the socio-organisational settings in which this knowledge finds its meaning.
\end{abstract}




\section{Des enjeux qui conduisent à renouveler les modes de production des connaissances agronomiques}

Depuis la dernière guerre mondiale, le développement agricole dans les pays industrialisés a largement bénéficié des efforts de la recherche agronomique, qui a beaucoup fait progresser la conduite des cultures. Depuis une dizaine d'années, l'agriculture est de plus en plus au centre de débats de société, lors de crises aiguës à propos de sécurité des aliments, mais aussi plus profondément à propos de ses fonctions au sein de l'espace rural. Dans ce contexte, les institutions de recherche, de formation et de conseil - les «systèmes de connaissance agricole » (Röling, 1988) doivent concevoir de nouveaux modes de collaboration entre agriculteurs, agents de développement et scientifiques, mais aussi renouveler les connaissances scientifiques à produire (Hubert et al., 2000). Mais si les dispositifs de collaboration entre chercheurs et acteurs, et plus largement les relations entre science et société, sont largement débattus, peu de travaux portent sur le contenu des connaissances, notamment agronomiques $^{1}$, à construire dans ces situations.

Notre hypothèse de travail ${ }^{2}$ est que des connaissances agronomiques peuvent émerger d'une synergie entre connaissances empiriques ${ }^{3}$ des praticiens et connaissances scientifiques. À propos de cette synergie, nous explicitons d'abord notre positionnement scientifique et brossons un rapide panorama des sources de connaissances empiriques et scientifiques disponibles. Puis nous explorons quatre modes de synergie permettant de combiner les deux types de connaissances. Dans chacune de ces deux parties, nous nous appuyons sur le cas des cultures de safran et de truffe, dont le développement bute sur une insuffisante maîtrise technique (Chazoule, 2001 ; Viard, 2001).

\section{Positionnement scientifique}

\section{Problématique : explorer la synergie entre connaissances scientifiques et empiriques}

Dans l'étude des systèmes agraires, les travaux sur les connaissances empiriques ${ }^{4}$ et les connaissances

\footnotetext{
${ }^{1}$ C'est-à-dire sur les processus biophysiques et sur la manière de les contrôler par des techniques culturales pour obtenir une production agricole.

${ }^{2}$ Cet article provient d'une recherche conduite autour de la relance de la culture de safran dans le Quercy (projet Inra PSDR Midi-Pyrénées).

${ }^{3} \mathrm{Au}$ sens de connaissances construites en accumulant des observations et des expériences et en les conceptualisant (Kolb, 1984).

${ }^{4}$ Nous utilisons ici le terme de connaissances empiriques plutôt que celui de connaissances "locales", afin de centrer
}

scientifiques sont souvent disjoints : les premières sont en effet souvent étudiées dans les systèmes à faible niveau d'intrants des pays en voie de développement, tandis que les secondes le sont davantage dans les pays industrialisés (Price, 2001). Les connaissances des producteurs présentent l'avantage d'être plus directement reliées aux questions des acteurs locaux, dont elles assurent confiance et participation (Zurayk et al., 2001). Par ailleurs, dans les pays en voie de développement, de nombreux travaux considèrent qu'elles risquent de disparaître, ce qui conduit à les extraire pour les conserver (par exemple, les Centres d'information et de connaissance autochtones de la Banque mondiale, 1998). Mais, dans la perspective située qui est la nôtre, une telle démarche nie l'aspect dynamique et contextuel de la cognition $^{5}$.

Ainsi, les deux mondes se rencontrent peu : les scientifiques et les agents de développement ont du mal à expliciter les connaissances des producteurs, tandis que les publications scientifiques sont souvent inaccessibles aux producteurs (Sumberg et al., 2003).

Nous excluons ici les situations où les connaissances empiriques sont vues comme un adversaire (cas lorsqu'une dynamique de «transfert de technologie » domine, selon Blaikie et al., 1997), au profit de situations où il semble utile de mobiliser différentes sources de connaissances, dont celles des producteurs. Nous cherchons à mobiliser les connaissances des producteurs, à la différence de Sumberg et al. (2003) qui, dans leur modèle "synergistic ", reposant sur une interaction constructive entre expérimentations scientifiques et expérimentations des producteurs, cherchent à intégrer ces derniers dans un processus de «recherche formelle». De plus, nous traitons du contenu des connaissances agronomiques, ce qui est original par rapport à la plupart des situations dans lesquelles sont associées différentes formes de connaissances : dans ces situations, des résultats importants ont été obtenus concernant les objectifs d'une action de développement et ses lignes générales de mise en œuvre, mais les aspects techniques sont rarement traités, ainsi que le constate Veiga (1999). La position originale adoptée dans ce texte est donc d'explorer la synergie ${ }^{6}$ entre connaissances empiriques et scientifiques ${ }^{7}$, et de voir en

notre propos sur le mode de production des connaissances, non sur leur origine.

5 Selon le paradigme de la Cognition/Action Située (Suchman, 1990), la connaissance est constitutive de l'action et ne peut donc être séparée d'une situation dans laquelle elle prend sens.

${ }^{6}$ Nous définissons la synergie comme une combinaison qui produit un résultat supérieur à la simple addition de ses composantes.

7 Cette dichotomie entre connaissances scientifiques et empiriques reste simpliste : les connaissances scientifiques peuvent comporter une part d'empirisme et, à l'inverse, les producteurs utilisent des connaissances d'origine scientifique. Dans la suite 


\begin{abstract}
Encadré 1. Le cas du safran
La culture du safran aurait été introduite dans le Quercy au XII siècle, puis abandonnée aux alentours de la révolution française. Seuls les bulbes ont parfois subsisté dans les jardins potagers, faisant plus partie de la tradition culinaire qu'agricole (Helfer, 2002). C'est pour faire revivre une culture oubliée, mais appartenant au patrimoine local, qu'une poignée de passionnés a imaginé de relancer la culture du safran en 1997. Cette relance a pris une dimension agricole et commerciale avec l'adhésion de la chambre d'agriculture du Lot et d'une coopérative, puis avec la fondation de l'Association des safraniers du Quercy en 1999 (actuellement une soixantaine de producteurs), et enfin avec les projets de Label Rouge et d'IGP.

Les volumes produits sont très faibles (4 kilos pour tout le Quercy, qui correspond à la surface de deux départements français), compte tenu des surfaces plantées (moins de 1 ha pour l'ensemble des producteurs) et de la nature des organes récoltés (les stigmates : partie terminale du pistil des fleurs). Mais le chiffre d'affaires est plus important du fait d'un prix de vente impressionnant (30€ le gramme en Quercy).

Aucune structure de recherche, de conseil ou d'enseignement n'est strictement dédiée au safran. En tant que plante aromatique, le safran rentre dans les activités de l'Institut technique des plantes aromatiques et médicinales (ITEIPMAI) et de l'Office national interprofessionnel des plantes aromatiques et médicinales (ONIPPAM), sans que ces organismes n'aient jusqu'à ce jour vraiment engagé d'actions en la matière. En 2003, à la demande de l'ASQ, une station expérimentale régionale s'est engagée sur le sujet. Enfin, rares sont les travaux scientifiques qui traitent de la conduite du safran (un seul article présent dans la base de données de l'Inra).
\end{abstract}

\title{
Encadré 2. Le cas de la truffe
}

Historiquement, la truffe est le produit d'une cueillette ; ce n'est qu'à partir de 1810 que les truffières naturelles ont été remplacées progressivement par des truffières cultivées. Malgré cette «domestication de la truffe », on assiste depuis un siècle à un déclin de la production, de 1200 tonnes vers 1880 à 20 tonnes dans les années 1990 (Byé et Chazoule, 1998).

On compte aujourd'hui environ 5000 ha plantés et 8000 à 10000 trufficulteurs. La plupart d'entre eux sont des « traditionnels ", c'est-à-dire qu'ils cueillent les truffes spontanées sur des truffières naturelles. Mais ce sont les «modernes », qui plantent et cultivent des arbres truffiers, qui assurent la majeure partie de la production (Olivier et al., 1996). Les rendements varient de 20 à $50 \mathrm{~kg} /$ ha et le prix de 750 à $1000 € / \mathrm{kg}$ (marché de Carpentras, 2003).

Il existe un foisonnement d'organisations, groupements de producteurs, syndicats, etc., au niveau local. Au niveau national, diverses organisations professionnelles ont été créées dans les années 1950 pour faire face au recul des quantités mises en marché, puis regroupées au sein de la Fédération française des trufficulteurs en 1996. Cet organisme coordonne notamment des recherches et des études sur la filière. Le Centre technique interprofessionnel des fruits et légumes est impliqué depuis plus de 20 ans avec de nombreuses truffières expérimentales et la rédaction d'ouvrages techniques (Ricard, 2003). L'Inra a conduit différentes études sur les conditions de développement du champignon (Callot, 1999) et mis au point la méthode de mycorhization de plants (Chevalier et Grente, 1979). Le Centre national d'expérimentation sur la truffe vise à coordonner les expérimentations menées par ces divers organismes. Pourtant, malgré un important dispositif de recherche-développement, comme en témoignent les nombreuses journées techniques et publications de vulgarisation, Olivier et al. (1996) considèrent que la France est en retard par rapport à d'autres pays producteurs, comme l'Italie.

quoi elle est source de nouvelles connaissances. Cela suppose de s'inscrire dans une démarche d'ingénierie des connaissances $^{8}$ et donc de dépasser l'aller-retour entre une approche ethnographique des savoirs empiriques et une agronomie qui chercherait à s'appuyer sur la pratique pour produire des connaissances pertinentes pour l'action.

\section{Démarche}

Nous nous appuyons sur deux études de cas, le safran et la truffe (Encadrés 1 et 2), productions singulières d'un point de vue agronomique (Encadré 3), pour lesquelles

du texte, nous garderons cependant ces termes pour mettre l'accent sur leur mode de production.

8 Au sens de l'activité qui consiste à expliciter et mettre en forme des connaissances provenant de différentes sources et non de la communauté scientifique du même nom (Teulier et Girard, 2001). les volumes produits sont bien en deçà des possibilités de débouchés : production en cours de relance et donc avec une faible surface cultivée pour le safran, en déclin pour la truffe. L'image de ces deux produits ne cesse de se valoriser auprès des consommateurs et leurs cours sont très élevés. Mieux maîtriser techniquement ces cultures devient un enjeu fort qui passe, selon nous, par une mise en synergie entre connaissances empiriques et scientifiques. Ces deux cas présentent un fort pouvoir explicatif dans le cadre de cet article : nous les avons utilisés dans un processus inductif d'abstraction (Mitchell, 1983) permettant d'en tirer des conclusions génériques, tout en les référant aux particularités du contexte, de la situation et des acteurs. Nos données proviennent :

- d'entretiens individuels avec des producteurs sur leurs pratiques et la manière dont ils les justifient (sources de connaissances, indicateurs qu'ils mobilisent...) : une trentaine de producteurs de 
Encadré 3. Bases agronomiques des cultures de safran et de truffe

- Le safran (Viard, 2001). C'est une plante à bulbe dont le cycle de développement se divise en trois grandes phases (Fig. 1). La floraison débute en septembre avec la germination des bourgeons végétatifs, l'émergence des feuilles, la croissance de la hampe florale et la maturation des fleurs. La phase végétative s'étend ensuite de décembre à mai avec la croissance et le développement des feuilles, le grossissement des bulbes-filles et la dégénérescence du bulbe-mère. Enfin, vient la phase de dormance (maiseptembre), avant le début d'un nouveau cycle. L'épice s'obtient par émondage des fleurs (Fig. 1). La conduite d'une safranière est à la fois annuelle (fertilisation et désherbage principalement) et pluriannuelle (arrachage et replantation des bulbes).

Le safran est une plante singulière au plan agronomique. C'est une plante pour partie souterraine, ce qui rend difficile l'appréciation de l'état du peuplement. Son cycle est à végétation inversée (développement végétatif de septembre à mai, après la floraison). Si chaque bulbe a un cycle annuel, la culture est pérenne puisque le peuplement végétal se multiplie de lui-même d'année en année. Enfin, l'objectif de production est très différent des autres cultures, car la partie récoltée est le stigmate (Fig. 1). - La truffe (Olivier et al., 1996). C'est un champignon souterrain, en symbiose avec un hôte. La culture repose sur la plantation d'arbres-hôtes (chêne, noisetier. . ) porteurs de mycélium truffier. Après une phase d'installation de la symbiose entre les racines de l'arbre et le champignon (6-10 ans), la truffière produit pendant 15 à 40 ans (Fig. 2). Conduire une truffière consiste essentiellement à piloter les arbres-hôtes et le milieu (plantation, taille des arbres pour contrôler la vigueur, irrigation, travail du sol, protection des arbres contre les maladies et les parasites). La récolte a lieu entre novembre et mars.

La truffe est, elle aussi, une plante singulière : champignon en symbiose, sa croissance répond à des processus très différents des cultures classiques. C'est une culture pérenne à durée de vie très longue.

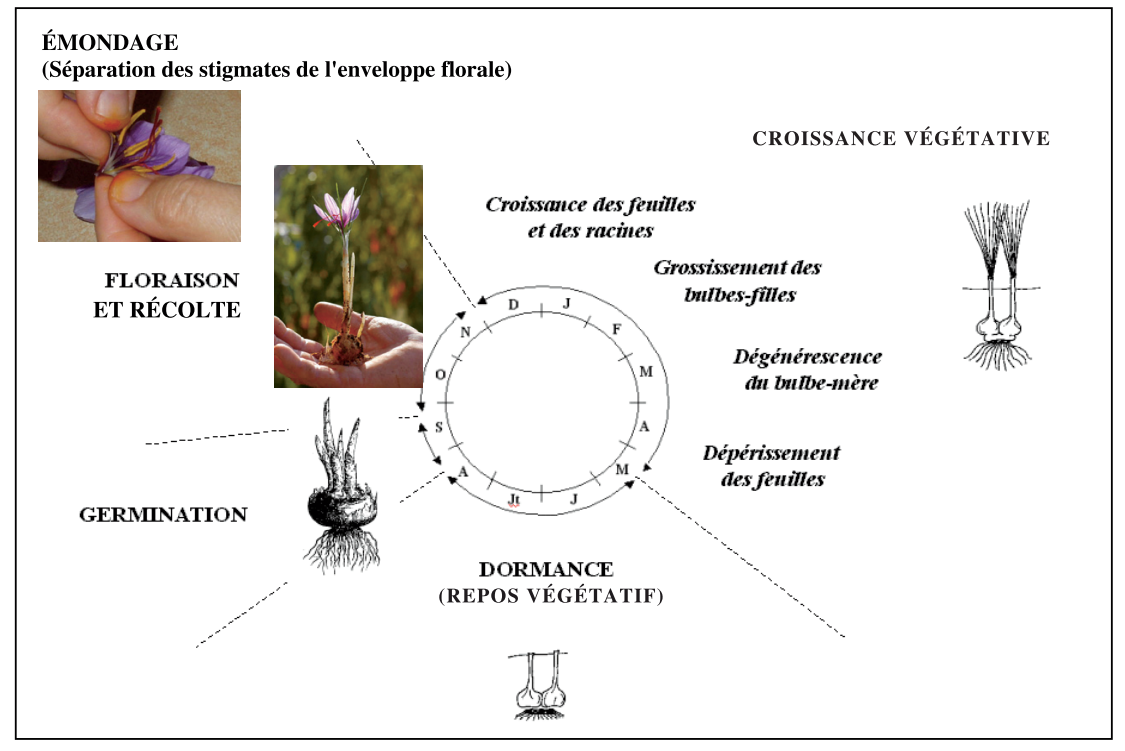

Fig. 1. Cycle biologique du safran (Crocus satious) (adapté de Viard, 2001).

l'Association des safraniers du Quercy (ASQ) ont ainsi été rencontrés en 2001 (Viard, 2001);

- du recueil de traces écrites : «cahiers d'observation », où certains producteurs notent leurs pratiques et observations sur leur culture, documents techniques produits par le conseiller de l'ASQ;

- des observations et/ou enregistrements de réunions entre producteurs : (i) réunions organisées par le conseiller de l'ASQ, en salle (bilans de campagne de l'ASQ en 2000 et 2001, groupe de travail de 1'ASQ sur le lancement d'une démarche qualité en 2001) ou sur le terrain (visite d'une safranière en avril 2001); (ii) session de formation sur la conduite de truffières organisée par la Fédération française de trufficulture, réunissant une quinzaine de conseillers techniques, eux-mêmes pour une grande partie trufficulteurs (juin 2002).
Partant d'une perspective située, nous accédons aux connaissances empiriques à la fois par les expressions de connaissances (discours individuels, écrits et discussions collectives) et par les pratiques, l'objectif étant d'expliciter les connaissances que les producteurs utilisent pour conduire leur culture, de comprendre la façon dont ils choisissent les modalités techniques et de faire exprimer les problèmes techniques rencontrés. Parallèlement, une analyse de la littérature scientifique et technique nous a permis de dresser un état des lieux des connaissances disponibles sur nos deux cas d'étude.

Notre analyse a consisté à confronter ces sources de données tout en mobilisant la théorie agronomique générale, afin de cerner différents modes possibles de combinaison entre connaissances empiriques et scientifiques. 


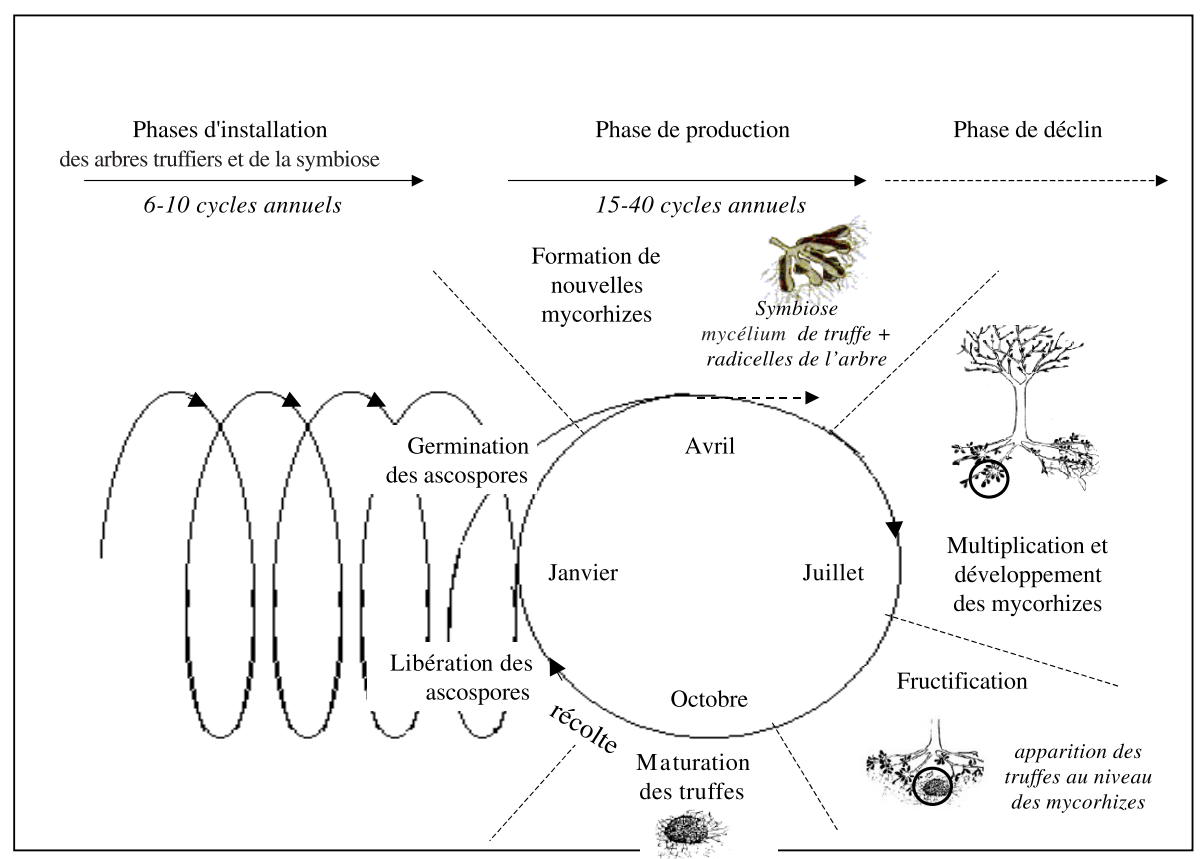

Fig. 2. Dynamique pluriannuelle et cycle annuel de la truffe.

\section{Deux cas d'étude : le safran et la truffe}

\section{Des enjeux techniques portés par des collectifs hétérogènes}

Des collectifs institués se sont créés pour répondre à des enjeux tels que relancer la production, construire ou soutenir une "filière agricole ", ou mettre en valeur un patrimoine local (Encadrés 1 et 2).

\section{Des collectifs hétérogènes. . .}

Ces collectifs sont extrêmement hétérogènes : les producteurs adhérents ont des activités professionnelles très diverses (par exemple, la moitié des safraniers de l'ASQ ne sont pas agriculteurs au sens statutaire, mais enseignants, retraités ou encore artisans). Ces cultures constituent ainsi pour certains une activité de diversification agricole, alors que pour d'autres c'est une façon de renouer avec l'activité agricole ou une opportunité pour se reconstruire un lien au terroir. De ce fait, leurs attentes par rapport à la culture sont très diverses : objectif de production pour certains (pour assurer un revenu, principal ou complémentaire, pour diversifier, pour mieux vendre d'autres produits dérivés, comme le sirop au safran); objectif de culture pour d'autres (pour protéger ou promouvoir un patrimoine historico-culturel, comme l'agrotourisme autour du safran ou la gestion du patrimoine foncier pour la truffe) ; objectif social pour d'autres encore (faire partie d'un groupe, par exemple).

\section{... avec un fort enjeu technique collectif}

La très grande variabilité de rendement, entre parcelles et entre années, atteste du manque de maîtrise technique de ces cultures : par exemple, pour le safran, le nombre de fleurs rapporté au nombre de bulbes initialement plantés peut passer de $0 \%$ à presque $200 \%$. Ainsi, même les producteurs les plus expérimentés se font surprendre par les résultats obtenus, car, comme le dit une productrice forte de l'expérience de 30 années de culture du safran : "On a beau faire la même chose, ça change. C'est l'énigme ${ }^{9}$.»

À un niveau collectif, cette variabilité de la production individuelle fragilise les filières et démarches collectives, qui ont du mal à faire des prévisions de production et donc à élaborer une stratégie commerciale, mais aussi à se projeter dans l'avenir en tant que collectif (recrutement d'un animateur, statut du collectif... ).

\section{Des enjeux de synergie entre connaissances empiriques et scientifiques}

La relance de ces productions et leur soutien passe donc par une meilleure maîtrise de la conduite de ces cultures. Pourtant, les différentes sources de connaissances ne permettent pas, de manière isolée, de répondre à cet enjeu.

\footnotetext{
9 Bilan de campagne de l'ASQ (décembre 2001). Les citations sont extraites de nos données.
} 


\section{Des connaissances empiriques très hétérogènes}

Certains producteurs cultivent safran ou truffe depuis de nombreuses années, voire plusieurs générations, et se sont forgés, au fil des ans, leur propre connaissance en matière de conduite de ces cultures. Certains vont même jusqu'à expérimenter par eux-mêmes ${ }^{10}$. Mais ces seules connaissances empiriques ne suffisent pas à atteindre l'enjeu technique collectif, d'une part parce qu'elles sont disparates, voire contradictoires, d'autre part parce qu'elles sont tacites et difficilement partageables.

\section{Des connaissances individuelles disparates, voire contradictoires}

Ainsi, en parlant des trufficulteurs, les conseillers constatent $\mathrm{qu}^{\prime}$ « on arrive à entendre tout et son contraire ». Par exemple, à propos du choix du meilleur terrain pour implanter une truffière (session de formation de juin 2002), certains producteurs considèrent que «la vigne, c'est le précédent idéal », tandis que d'autres avancent que «chez nous, c'est la luzerne, ça nettoie». Parmi ceux qui privilégient les «terrains ingrats », certains considèrent que la présence de cailloux est favorable à la culture de la truffe, tandis que d'autres justifient ce choix par défaut, parce qu'aucune autre espèce n'y pousse. D'autres enfin argumentent qu'ils ont toujours vu les truffières sur les anciennes terres à vigne, mais sans que cette culture ne soit forcément considérée comme un bon précédent agronomique ${ }^{11}$. Face à ces connaissances où se mêlent étroitement expérience individuelle, histoire locale et connaissances techniques transposées à partir d'autres cultures, lieux ou époques, il n'est guère étonnant que des contradictions apparaissent entre producteurs.

\section{Des connaissances tacites et difficilement partageables}

De plus, les connaissances mobilisées par les producteurs sont tacites (Nonaka et Takeuchi, 1997) et donc difficilement partageables. Ainsi, les producteurs font largement appel à leur propre perception de l'état de la culture au travers d'indicateurs qualitatifs. Par exemple, la couleur de la feuille de safran en sortie d'hiver est un indicateur utilisé pour décider de la date d'un désherbage chimique (visite de terrain d'avril 2001). Mais l'échelle de valeur pour apprécier ce stade reste propre à chacun.

\footnotetext{
$10 \mathrm{Au}$ sens des expérimentations exploratoires de Schön (1994), c'est-à-dire qui « consistent à s'amuser à explorer pour acquérir une certaine connaissance des choses ».

${ }^{11}$ Le développement des truffières sur les anciennes terres à vigne est notamment dû au fait que ces terrains étaient disponibles après la destruction des vignes par le phylloxéra à la fin du XIXe siècle.
}

La difficulté à partager les connaissances est accrue par la diversité des origines socioprofessionnelles des producteurs, et donc des connaissances techniques et des langages utilisés. Ainsi, l'unité classiquement utilisée pour caractériser la fertilisation (unités NPK ramenées à l'hectare) n'est pas connue par les producteurs non agriculteurs. Les unités utilisées par certains producteurs, par exemple l'épaisseur de fumier épandu ou le nombre de seaux déversés, rendent difficile tout échange entre eux (Viard, 2001).

\section{Des connaissances scientifiques et techniques inopérantes}

Les connaissances scientifiques ne portent pas sur tout le cycle cultural ou toutes les opérations techniques. Par exemple, pour le safran, des spécialistes des plantes à bulbes ont identifié les facteurs jouant sur la sortie de dormance et le développement de la plante (Le Nard et Biot, 1994), alors que d'autres ont étudié la relation entre calibre du bulbe à la plantation et rendement en fleurs et bulbilles (De Mastro et Ruta, 1993). Mais la relation entre fertilisation et calibre du bulbe, essentielle pour les producteurs, reste méconnue. Ce qui illustre bien la difficulté de dialogue entre connaissances scientifiques et point de vue des producteurs, comme l'a constaté Veiga (1999) sur des fronts pionniers au Brésil.

Les connaissances scientifiques sont par ailleurs disciplinaires et morcelées. Ce morcellement n'est en fait qu'un reflet de la compartimentation des disciplines scientifiques. Par exemple, sur la truffe, les premières recherches ont été portées par des mycologues et des botanistes, préoccupés de la description, la classification et la reproduction du champignon (Chazoule, 2001). Les recherches récentes ont porté majoritairement dans deux directions : la symbiose entre le mycélium de la truffe et la racine de l'arbre-hôte par les physiologistes, et l'effet des conditions physiques et biotiques du sol sur la croissance du champignon par les pédologues (Callot, 1999).

Ces connaissances scientifiques sont donc d'abord issues de disciplines scientifiques fondamentales qui cherchent avant tout à améliorer la compréhension de processus biophysiques. L'agronomie, en tant que science de l'action (Papy, 2000), n'a pas travaillé sur ces espèces, ce qui n'est guère étonnant puisque, historiquement, ces deux produits relevaient de la cueillette.

Il est donc illusoire de chercher à formaliser un itinéraire technique, c'est-à-dire une combinaison logique et ordonnée de techniques qui permettent de contrôler le milieu et d'en tirer une production donnée (Sebillotte, 1974), en juxtaposant ces connaissances scientifiques fondamentales. Il faudrait en effet connaître les interactions entre les différentes opérations culturales appliquées à une même culture, et entre le milieu et le peuplement 
végétal, tout au long du cycle cultural, ce qui est loin d'être le cas pour ces deux cultures.

Il existe par ailleurs de nombreux manuels techniques sur les modes de gestion de ces cultures - documents anciens (publications de sociétés savantes du XIXe siècle : Pradel, 1914; Chappellier, 1896) ou guides proposés par des instituts techniques. Mais ces documents restent généralement vagues sur les modalités concrètes à mettre en œuvre. Par exemple, pour la truffe, Ricard (2003) indique que «les sols les plus favorables à une entrée rapide en production semblent être les terrains ayant une bonne structure et une prépondérance de matières organiques évoluées... mais il n'est pas possible à ce jour de donner des valeurs seuils en ce qui concerne la quantité et la qualité de la matière organique ».

\section{Différents modes de synergie entre connaissances empiriques et scientifiques}

Les différentes sources de connaissances sur le safran et la truffe, prises isolément, ne permettant pas de répondre aux enjeux techniques collectifs, nous identifions quatre modes de synergie pour y répondre.

\section{Mode 1 : traduire des connaissances empiriques en questions scientifiques pertinentes pour l'action}

Ce premier mode de synergie consiste à traduire des connaissances empiriques, explicitées par une démarche de type ethnographique, en questions scientifiques pertinentes pour l'action. Déjà pratiquée dans certains courants de l'agronomie, une telle traduction reste atypique par rapport aux démarches classiques, qui procèdent plutôt en comblant systématiquement les lacunes de connaissances sur le fonctionnement du peuplement végétal.

On peut, par exemple, déduire des différents modes de désherbage du safran les objets sur lesquels devraient porter les connaissances agronomiques à produire :

- les amateurs désherbent manuellement, souvent au jour le jour : ils ont besoin de connaissances sur les adventices pour les distinguer du safran (dont les feuilles ressemblent à celles des graminées) quel que soit leur stade de développement;

- à l'inverse, les professionnels ${ }^{12}$ procèdent généralement à un désherbage chimique unique dans l'année. Ils cherchent donc à connaître les produits actifs

\footnotetext{
12 «Amateurs » et «professionnels» sont des qualificatifs que se donnent les producteurs eux-mêmes au sein de l'Association des safraniers du Quercy, pour distinguer ceux qui cultivent le safran par passion de ceux qui souhaitent en tirer un revenu.
}

(désherbant sélectif ou total), les avantages et inconvénients de chacun et leurs conditions d'application. Ils doivent également savoir identifier le stade où le désherbage est le plus efficace pour diminuer la compétition entre adventices et culture.

Adventices au fil de l'année pour les uns ou, au contraire, stade optimal de désherbage pour les autres, les objets sur lesquels doivent porter les connaissances agronomiques à produire ne sont donc pas les mêmes. Dans un cas, ils renvoient à des connaissances en biologie et en botanique, sur les cycles de développement des différentes adventices. Dans l'autre, ils renvoient à des connaissances sur les besoins du safran en lumière, éléments minéraux et eau, sur les facteurs de croissance limitants et sur la concurrence des adventives, et ce pour les différentes phases du cycle cultural.

\section{Mode 2 : combiner connaissances heuristiques des producteurs et connaissances agronomiques sur les mécanismes biologiques sous-jacents}

Le deuxième mode consiste à fonder scientifiquement les connaissances empiriques, en mobilisant la capacité explicative de l'agronomie dans une démarche mécaniste. Les producteurs se construisent en effet des connaissances heuristiques, c'est-à-dire qui les aident à rechercher des solutions pour agir, à partir de la récurrence d'expériences similaires et de leur représentation personnelle des processus biologiques. Mais ces connaissances restent superficielles : elles reposent sur des associations entre états du monde (par exemple, les producteurs expérimentés de truffe, partant de la constatation qu'il y a souvent des grosses truffes aux endroits qui ne sont pas labourés, ont formalisé progressivement une règle selon laquelle il faut faire un «mauvais travail du sol »), mais ne les expliquent pas, ce qui n'est pas sans danger dans certaines situations.

Il peut donc être extrêmement utile d'identifier, parmi ces connaissances heuristiques, lesquelles sont fondées d'un point de vue scientifique et peuvent donc servir de référence technique collective. Par exemple, l'expression "bulbe fleureux», que l'on trouve dans des documents anciens, comme Ursat (1913), ou les observations des safraniers eux-mêmes («plus le bulbe est gros, plus il risque de faire plusieurs fleurs $\left.{ }^{13} »\right)$, ont pu être utilement rapprochées de la relation démontrée entre le nombre de fleurs et le calibre du bulbe (Viard, 2001, Annexe 3).

En d'autres cas, de tels rapprochements sont impossibles, faute de connaissances spécifiques au safran ou à la truffe. Pour la fertilisation du safran, par exemple, certains safraniers s'appuient sur l'hypothèse d'une compétition entre l'appareil végétatif et le bulbe : certains en déduisent que, pour limiter la croissance des feuilles,

\footnotetext{
13 Bilan de campagne de l'ASQ (décembre, 2000).
} 
il faut apporter beaucoup de potassium et de phosphore, et/ou peu d'azote ${ }^{14}$; d'autres considèrent que «c'est la végétation qui fait le bulbe » et qu'il faut donc apporter une dose d'azote forte ${ }^{15}$. Ainsi, ces connaissances heuristiques - ici sur «ce qui fait le bulbe » - conduisent à une diversité de pratiques et, globalement, à un excès de fertilisation $^{16}$ (Viard, 2001).

L'agronomie, en révélant les mécanismes sous-jacents aux objets de ces connaissances heuristiques, permet de les contextualiser, même si elles sont apparemment contradictoires. Pour reprendre l'exemple de « ce qui fait le bulbe » pour le safran, les écophysiologistes ont montré qu'à un instant donné, il existe une compétition entre tous les organes (théorie "source-puits », Warren-Wilson, 1972) et que la croissance foliaire est en concurrence avec celle du bulbe. Mais ces mêmes écophysiologistes ont aussi montré que la production de matière carbonée, principal élément constitutif du bulbe, est fonction de la surface totale des feuilles (Monteith, 1977). Sur le long terme, un appareil foliaire important est donc favorable au grossissement du bulbe. On peut ainsi considérer que les deux arguments des producteurs, en apparence contradictoires, sont valides à condition de les positionner sur le cycle de développement du safran : priorité à la production foliaire en début de cycle, puis à l'affectation des ressources carbonées au bulbe quelques semaines avant floraison. En terme de fertilisation, cela revient à préconiser un fractionnement de la fertilisation, avec un apport riche en azote en début de cycle, riche en phosphore et potassium ensuite (Chaux et Foury, 1994). Mais, pour préciser des règles de pilotage de la fertilisation, il faudrait définir plus précisément l'évolution des besoins en éléments minéraux au cours du temps, et calculer les doses nécessaires à chaque phase par des méthodes de bilan.

\section{Mode 3 : relier les indicateurs des producteurs à des paramètres agronomiques reproductibles}

Le troisième mode de synergie renvoie à une approche empirique qui consiste à faire exprimer et formaliser les indicateurs des producteurs expérimentés, puis à vérifier leur fiabilité par une analyse de leur corrélation statistique avec des paramètres agronomiques mesurables.

Dans ces conditions, il s'avère particulièrement important de disposer d'indicateurs indirects d'état du peuplement. Partiellement ou totalement souterrains,

\footnotetext{
14 À l'extrême, 0 unité $\mathrm{N}$ et 560 unités PK pour un producteur enquêté.

15 À l'extrême, 740 unités $\mathrm{N}$ et 0 unité $\mathrm{PK}$ pour un autre producteur enquêté.

16 À titre de comparaison, l'ordre de grandeur des doses apportées sur blé est de 200 unités N, 60 P, 60 K pour des quantités de matière sèche produite par hectare bien supérieures.
}

le safran ou la truffe sont regardés avec un respect distancié par les producteurs ${ }^{17}$ qui, de ce fait, manquent d'informations sur l'état du peuplement. Par exemple, pour les safraniers expérimentés, "il ne faut pas désherber ${ }^{18}$ avant la mort du safran », indicateur empirique qui correspond à l'arrêt du fonctionnement photosynthétique du feuillage, c'est-à-dire à l'entrée en dormance de la plante à la fin du cycle végétatif (Fig. 1). De tels indicateurs qualitatifs reposent largement sur des perceptions tacites et difficilement partageables. D'autant que des divergences existent sur la façon d'évaluer cet indicateur : certains producteurs estiment qu'on peut traiter dès que les feuilles commencent à jaunir, tandis que d'autres préfèrent attendre la disparition complète du feuillage.

Dans le cas de la truffe, les producteurs observent la vigueur des arbres-hôtes pour réaliser certaines interventions techniques (par exemple, " garrotter $^{19}$ les arbres trop vigoureux »), sans expliciter vraiment comment ils évaluent cet indicateur subjectif.

On s'attachera ici à rechercher des échelles de valeur mesurables, donc reproductibles. Ainsi, les producteurs de tomates sous serre utilisent la « vigueur » des plantes pour adapter la taille de celles-ci ou gérer le climat de la serre. Navarrete et al. (1997) ont montré que les indicateurs utilisés par certains maraîchers expérimentés, bien que peu codifiés (port et couleur du feuillage, épaisseur de la tige. . . ), sont relativement fiables, et qu'il existe une bonne corrélation entre des notations de vigueur réalisées par des producteurs expérimentés et des mesures de diamètre de tige. La vigueur peut donc être estimée par cette mesure, facilement accessible aux non-initiés.

Des dispositifs comparables pourraient être mis en place pour le safran et pour la truffe. Dans le cas du safran, il faudrait, par exemple, définir expérimentalement à partir de quel stade de son développement la plante peut subir un traitement chimique sans pénaliser la culture. De plus, si le jaunissement de la feuille de safran se révèle un bon indicateur pour choisir une date de traitement, il faudrait proposer une palette de couleurs pour qualifier objectivement la couleur des feuilles (semblable à celle déjà utilisée par les arboriculteurs pour la récolte des fruits).

Dans le cas de la truffe, le problème est plus complexe, car la «vigueur des arbres » est un indicateur indirect de la croissance des truffes : en agissant sur l'arbre, les producteurs n'interviennent qu'indirectement sur le peuplement de truffe, en modifiant le système racinaire et donc la symbiose arbre-champignon. Si la vigueur d'un arbre peut être approchée par le diamètre du tronc ou des ramifications - comme l'a fait Champagnol (1984) pour

\footnotetext{
17 En parlant des bulbes, les safraniers disent fréquemment $\mathrm{qu}^{\prime}$ « il ne faut pas les déranger $» .$.

18 Sous-entendu par des moyens chimiques.

19 Faire une ligature sur certaines branches pour limiter la circulation de la sève.
} 
la vigne -, il faudrait donc aussi analyser la corrélation entre vigueur de l'arbre et rendement en truffe.

\section{Mode 4 : organiser une analogie entre cultures sur la base d'arguments scientifiques}

En l'absence de connaissances spécifiques à leur culture, les producteurs de truffe et de safran conduisent également leurs cultures par analogie avec d'autres cultures mieux connues. L'analogie peut toutefois s'avérer dangereuse lorsqu'elle incite à transposer entre objets classés ensemble à tort (Stengers et Bensaude-Vincent, 2003) : il faut donc s'appuyer sur un argument scientifique pour rapprocher des plantes et cerner ce qui, parmi les connaissances disponibles, peut être transposable d'une plante à l'autre. On retrouve ici la notion de «plante-modèle», utilisée en agronomie pour transférer des connaissances élaborées sur une espèce à d'autres espèces (Fleury, 1994).

Par exemple, les safraniers s'appuient, pour expliquer leurs pratiques de fertilisation (Viard, 2001), sur des plantes très diverses qu'ils considèrent comme analogues du point de vue soit botanique (1), soit de leurs exigences en minéraux (2). En mobilisant des arguments scientifiques, il est possible d'évaluer ces analogies empiriques et de les rendre plus pertinentes :

1. L'analogie botanique avec la jacinthe et la colchique, c'est-à-dire avec d'autres plantes à bulbe, renvoie à la classification phylogénétique ${ }^{20}$. Rigoureusement, celle-ci désignerait plutôt l'iris ou le glaïeul comme plante-modèle pour le safran (Viard, 2001). Cependant, ces espèces proches (celles utilisées par les producteurs comme celles désignées par la classification phylogénétique) n'ont pas forcément le même cycle de développement : la jacinthe fleurit, par exemple, en mars et a donc un cycle de développement très différent de celui du safran, ce qui rend l'analogie dangereuse pour raisonner la fertilisation.

2. L'analogie physiologique avec le tabac et les céréales repose sur l'hypothèse que ces espèces ont les mêmes exigences en minéraux. Ce faisant, les producteurs risquent de choisir des doses d'engrais erronées parce qu'ils rapprochent :

- une espèce à bulbe et des espèces sans bulbe, dont la répartition de la matière sèche est très différente (ratio entre appareils végétatifs aérien et souterrain élevé pour le tabac, faible pour le safran);

- une culture pérenne (où la plante remobilise des éléments minéraux stockés d'une année à l'autre) et des cultures annuelles (où la seule source est le sol), qui ont des cinétiques de mobilisation des éléments minéraux très différentes;

${ }^{20}$ Qui rassemble les espèces selon leur proximité génétique.
- des espèces cultivées pour les fleurs (safran), les feuilles (tabac) ou les grains (céréales), alors que les besoins en azote, phosphore et potassium des différentes catégories d'organes sont très différents (Chaux et Foury, 1994).

On peut rechercher des plantes-modèles diverses suivant les phases du cycle et les composantes du rendement, comme l'a fait Fleury (1994) pour la pomme de terre. Dans le cas du safran, le rendement est fonction du nombre et du calibre des bulbes, et du nombre de fleurs par bulbe. Pour identifier les facteurs influençant le calibre et le nombre de bulbes de safran, les plantes cultivées pour leurs bulbes (ail, oignon, échalote...) constituent donc de bonnes plantes-modèles. On rejoint là le choix empirique des producteurs, tout en identifiant une piste pour cerner scientifiquement les doses et les dates d'apport de l'engrais. Par contre, pour la détermination du nombre de fleurs, il faudrait connaître l'effet de la fertilisation sur l'initiation florale, par exemple à partir d'une plante-modèle en horticulture ornementale.

\section{Discussion}

\section{Quelle(s) combinaison(s) entre connaissances empiriques et scientifiques?}

Les modes de synergie proposés consistent : à traduire des connaissances empiriques en questions scientifiques pertinentes pour l'action (mode 1); à appliquer des cadres théoriques généraux afin d'en déduire des connaissances mécanistes spécifiques au cas étudié (mode 2); à analyser statistiquement la corrélation entre indicateurs empiriques et paramètres mesurables (mode 3) ; à transposer des connaissances d'une culture à une autre à partir d'une analogie scientifiquement fondée (mode 4).

Étudier les pratiques des agriculteurs pour construire des questions scientifiques (mode 1) est un principe maintenant classique dans les courants des sciences agronomiques tournés vers l'action. De même, certains travaux cherchent à évaluer les connaissances empiriques par rapport aux connaissances scientifiques existant sur le même sujet (mode 3). Par exemple, Walker et al. (1999) ont montré que les connaissances empiriques avaient la même capacité que les techniques de laboratoire pour discriminer la valeur nutritive d'arbustes fourragers au Népal; de même, Barzman et al. (1996) ont vérifié le bienfondé scientifique de connaissances empiriques de lutte biologique contre les infestations d'insectes sur des citronniers au Vietnam. Néanmoins, dans une perspective 
descriptive plus qu'opérationnelle, ces travaux cherchent avant tout à vérifier scientifiquement les connaissances empiriques, sans vraiment les combiner.

\section{Connaissances spécifiques et génériques, méthodes et niveaux de généricité}

Les modes de synergie que nous avons proposés procèdent par abstraction à partir des pratiques des producteurs, ainsi que de leurs connaissances personnelles et contextuelles. Ces connaissances doivent alors être explicitées, notamment celles des producteurs les plus expérimentés : un certain nombre d'outils et de techniques existent pour cela (McGraw et Harbison-Briggs, 1989; Gaines et Shaw, 1993), dont certains inspirés de méthodes ethnographiques (Benfer et Furbee, 1990 ; Price, 2001). Mais appliquer de telles méthodes à chacun des producteurs et juxtaposer les connaissances ainsi acquises, comme cela est proposé par exemple par Sinclair et Walker (1998), conduit inévitablement à des répétitions et des ambiguïtés, comme le notent ces auteurs.

Or, répondre à un enjeu technique collectif ne saurait se fonder sur chacune des connaissances personnelles et spécifiques des producteurs, des pratiques particulières et des manières personnelles de faire; les objets de gestion, les indicateurs utilisés pour agir et les raisonnements tenus sont plus utiles en la matière.

Notre théorisation des connaissances empiriques permet alors de les rendre explicites, génériques, et partageables, à l'inverse, par exemple, de Zurayk et al. (2001) qui mobilisent des connaissances pédologiques et agroécologiques empiriques pour établir des cartes de potentialités d'une région du Liban.

Les connaissances à construire résultent alors d'un compromis subtil entre (i) une contextualisation excessive qui conduit à une redondance, des ambiguïtés et cache les connaissances ayant un potentiel générique, et (ii) une complète dé-contextualisation qui ferait perdre tout sens aux indicateurs et objets utilisés pour agir. Pour «capturer le contexte d'une connaissance », Sinclair et Walker (1998) proposent de modéliser le contexte d'énonciation de ces connaissances (la source, les conditions de validité et les relations hiérarchiques entre concepts). Mais ils ne proposent rien pour rendre compte du fait que la connaissance est constitutive de l'action et ne peut être séparée d'une situation dans laquelle elle prend sens. Afin de relier les connaissances exprimées à un contexte non pas seulement d'énonciation, mais d'action, il faut pouvoir les exprimer par rapport à une tâche à accomplir ou un contexte de référence. Nous rejoignons ici certains travaux, comme ceux de Cerf et al. (1998) qui, à propos de la tâche de démarrage du travail du sol au printemps, ont identifié par des méthodes de psychologie expérimentale les indicateurs d'états du sol partagés entre agriculteurs.

\section{Dispositifs socio-organisationnels, compétences des animateurs et modes d'apprentissage collectif}

Jusqu'à présent, notre approche a volontairement écarté des questions plus sociales et organisationnelles. Cependant, le principe d'inséparabilité entre savoirs et relations d'Hatchuel (2000) conduit à étudier aussi les modes d'organisation, de coordination et les systèmes de relations entre acteurs, dans lesquels prennent sens ces connaissances. La question se pose alors des dispositifs associant chercheurs, producteurs et conseillers. Cela suppose notamment de s'interroger sur les modes d'apprentissage collectif qui, selon Ingham (1994), sont de deux types :

- un mode "expérimental », consistant à provoquer des observations, par exemple en organisant des "pasture walks ", réunions entre producteurs sur le terrain (Hassanein et Kloppenburg, 1995) permettant des échanges spontanés de connaissances expérientielles. Les séances collectives d'émondage du safran, en favorisant les discussions entre safraniers sur les critères de qualité, relèvent de ce mode. Mais, dans ces groupes hétérogènes, un tel mode expérimental se heurte rapidement aux écarts « entre les bases de connaissances [... qui] limite[nt] les possibilités d'apprentissage » (Ingham, 1994). De tels écarts peuvent être reliés au manque de "grounding references », c'està-dire de termes permettant d'identifier rapidement et de façon fiable des objets lors d'échanges (Clark et Brennan, 1991);

- un mode "cognitif», comme essaie de l'organiser le conseiller de l'ASQ en cherchant à rationaliser des indicateurs. Ce mode renvoie directement vers la formalisation de connaissances empiriques et scientifiques combinées, comme nous avons pu l'explorer dans ce texte.

Ce sont donc deux modes complémentaires d'apprentissage que doivent favoriser les animateurs de ces collectifs. Mais la plupart d'entre eux sont avant tout formés pour animer des groupes d'agriculteurs, c'est-à-dire des groupes de pairs (comme «les groupes professionnels locaux » de Darré, 1988), et se trouvent démunis face à des groupes plus hétérogènes. Il s'agit alors de réinventer de nouvelles pratiques et de redéfinir un nouveau métier : ces situations où le développement territorial doit être pris en compte dans le développement agricole renvoient aux questions d'acquisition et de transmission des compétences nécessaires pour combiner différentes formes de connaissances, notamment à l'ingénierie de la formation (Girard et al., 2004). 


\section{Conclusion : des situations atypiques, mais des questions génériques}

Les deux études de cas mobilisées peuvent paraître atypiques. Cependant, dans le contexte socioéconomique actuel, elles représentent des situations qui risquent de se rencontrer également sur des cultures très connues, dès lors que l'on cherche à modifier les modes de culture pour des questions d'environnement, de santé... De plus, d'un point de vue épistémologique, faut-il considérer que la recherche agronomique doit toujours reproduire le même schéma de production de connaissances, commençant par une compréhension fine des processus écophysiologiques? Au contraire, l'évolution rapide des enjeux socioéconomiques auxquels est confrontée l'agriculture, leur complexité grandissante, leur élargissement au monde rural et non plus seulement agricole, poussent à diversifier les modes de production de connaissances, requérant de la recherche agronomique des réponses rapides, évolutives, sans qu'il soit toujours possible d'attendre que les mécanismes biologiques sousjacents soient parfaitement connus. Car il s'agit de produire des connaissances génériques tout en s'impliquant dans des situations spécifiques, par la mise en interaction de différentes sources de connaissances. Aujourd'hui, cet enjeu concerne toutes les sciences qui prétendent traiter en partenariat les problèmes complexes d'environnement et de gestion des ressources naturelles.

\section{Références}

Banque mondiale, 1998. Connaissances autochtones pour le développement. Un cadre pour l'action.

Barzman, M.S., Mills, N.J., Nguyen, T.T.C., 1996. Traditional knowledge and rationale for weaver ant husbandry in the Mekong Delta of Vietnam, Agriculture and Human Values, 13, 4, 2-9.

Benfer, R.A., Furbee, L., 1990. Knowledge acquisition: lessons from anthropology, AI applications, 4, 3, 19-26.

Blaikie, P., Brown, P., Stocking, M., Tang, L., Dixon, P., Sillitoe, P., 1997. Knowledge in action: local knowledge as a development resource and barriers to its incorporation in natural resource research and development, Agricultural systems, 55, 2, 217-237.

Byé, P., Chazoule, C., 1998. Production, protection et profession truffières, in Byé, P. (Ed), Domestiquer le végétal. Construction et appropriation des techniques, Montpellier, INRA-CTESI, 115-132.

Callot, G., 1999. La Truffe, la terre, la vie, Paris, INRA Éditions.

Cerf, M., Papy, F., Angevin, F., 1998. Are farmers expert at identifying workable days for tillage?, Agronomie, 18, 45-59.

Champagnol, F., 1984. Éléments de physiologie de la vigne et de viticulture générale, Montpellier, Dehan Press.

Chappellier, M.P., 1896. Compte rendu des cultures de Safran faites à la commanderie (Loiret), Bulletin de la société botanique de France.

Chaux, C., Foury, C., 1994. Productions légumières. T. 1. Généralités, Paris, Lavoisier.
Chazoule, C., 2001. Les Processus d'innovation en agriculture. Étude comparative à partir de deux mises en culture, les cas de la trufficulture et de l'arboriculture fruitière. Thèse, ENSAM, Montpellier.

Chevalier, G., Grente, J., 1979. Application pratique de la symbiose ectomycorhizienne : production à grande échelle de plants mycorhizés par la truffe, Mushroom Science, 10, 2, 483-505.

Clark, H.H., Brennan, S.E., 1991. Grounding in communication, in Resnick, L.B., Levine, R.M., Teasley, S.D. (Eds), Perspectives on socially shared cognition, Washington, American Psychological Association, 127-149.

Darré, J.-P., 1988. Production de diversité et production de connaissance, in Jollivet, M. (Ed.), Pour une agriculture diversifiée. Arguments, questions, recherches, Paris, L'Harmattan, 141-147.

De Mastro, G., Ruta, C., 1993. Effect of corm size at planting time on the corm enlargment and flowers production of saffron (Crocus Satious L.), International Symposium on Medicinal and Aromatic Plants, Israël, 22-25 mars 1993, 512-517.

Fleury, A., 1994. La notion de plante modèle, in Combe, L., Picard, D. (Eds), Un point sur l'élaboration du rendement des principales cultures annuelles, Paris, INRA Éditions, 7-27.

Gaines, B.R., Shaw, M.L.G., 1993. Knowledge acquisition tools based on personal construct psychology, Knowledge Engineering Review, 8, 1, 49-85.

Girard, N., Albaladejo, C., Labatut, J., 2004. From farm advisory work to rural facilitation: Facilitation practices and learning modes. The case of a saffron association in South-West France, Workshop "Knowing and Learning" du congrès IFSA, avril 2004, Vila Real (Portugal).

Hassanein, N., Kloppenburg, J.R., 1995. Where the grass grows again: knowledge exchange in the sustainable agriculture movement, Rural Sociology, 60, 4, 721-740.

Hatchuel, A., 2000. Quel horizon pour les sciences de gestion? Vers une théorie de l'action collective, in David, A., Hatchuel, A., Laufer, R. (Eds), Les Nouvelles Fondations des sciences de gestion, Paris Vuibert.

Helfer, S., 2002. Contribution d'une approche ethnohistorique à la relance du safran dans le Quercy. Mémoire de licence, Université de Lausanne.

Hubert, B., Ison, R.L., Röling, N., 2000. The "problematique" with respect to industrialised-country agriculture, in LEARN Group (Eds), Cow up a Tree. Knowing and Learning for Change in Agriculture. Case Studies from Industrialised Countries, Paris, INRA Éditions, 13-29.

Ingham, M., 1994. L'apprentissage organisationnel dans les coopérations, Revue française de gestion, 97, 105-121.

Kolb, D.A., 1984. Experiential learning: experience as a source of learning and development, New Jersey, Prentice Hall.

Le Nard, M., Biot, E., 1994. Essais de maîtrise de la floraison du safran (Crocus Sativus L.), Rivista italiana EPPOS, numéro spécial $12^{\text {es }}$ journées Huiles essentielles, 289-293.

Mcgraw, K.L., Harbison-Briggs, K, 1989. Knowledge acquisition: principles and guidelines, New Jersey, Prentice Hall.

Mitchell, J.C., 1983. Analyse de cas et de situation, The Sociological Review, 31, 2, 187-211 (traduction française de J.-P. Darré et V. Ménager).

Monteith, J.L., 1977. Climate and the efficiency of crop production in Britain, Phil. Trans. R. Soc., 281, 277-294.

Navarrete, M., Jeannequin, B., Sebillotte, M., 1997. Vigour of greenhouse tomato plants (Lycopersicon esculentum Mill.): Analysis of the criteria used by growers and search for objective criteria, Journal of Horticultural Science, 72, 821-829. 
Nonaka, I., Takeuchi, H., 1997. La Connaissance créatrice. La dynamique de l'entreprise apprenante, Bruxelles, De Boeck Université.

Olivier, J.-M., Savignac, J.-C., Sourzat, P., 1996. Truffe et trufficulture, Périgueux, Fanlac.

Papy, F., 2000. Farm models and decision support: a summary review, in Colin, J.P., Crawford, E.W. (Eds), Research on Agricultural Systems: Accomplishments, Perspectives and Issues, New York, Nova Science Publishers, 89-107.

Pradel, L., 1914. Manuel de trufficulture, guide pratique, Paris, Librairie J.-B. Baillière et fils.

Price, L.L., 2001. Demystifying farmers' entomological and pest management knowledge: a methodology for assessing the impacts on knowledge from IPM-FFS and NES interventions, Agriculture and Human Values, 18, 153-176.

Ricard, J.-M., 2003. La Truffe. Guide technique de trufficulture, Paris, CTIFL.

Röling, N., 1988. Extension Science. Information Systems in Agricultural Development, Cambridge, Cambridge University Press.

Schön, D.A., 1994. Le Praticien réflexif. À la recherche du savoir caché dans l'agir professionnel, Montréal, Les Éditions Logiques.

Sebillotte, M., 1974. Agronomie et agriculture. Essai d'analyse des tâches de l'agronome, Cahiers ORSTOM, 24, 3-25.

Sinclair, F.L., Walker, D.H., 1998. Acquiring qualitative knowledge about complex agrosystems. Part 1: Representation as natural language, Agricultural systems, 56, 3, 341-363.

Stengers, I., Bensaude-Vincent, B., 2003. 100 mots pour penser les sciences, Paris, Le Seuil.

Suchman, L, 1990. Plans d'action : problèmes de représentation de la pratique en sciences cognitives, Raisons pratiques 1, Les formes de l'action, 149-170.
Sumberg, J., Okali, C., Reece, D., 2003. Agricultural research in the face of diversity, local knowledge and the participation imperative: theoretical considerations, Agricultural systems, 76, 2, 739-753.

Teulier, R., Girard, N., 2001. Des connaissances pour l'action dans les organisations. Quelle ingénierie des connaissances pour assister l'activité, Journées Ingénierie des connaissances IC'2001, Grenoble, juin 2001, 253-272.

Ursat, J., 1913. Le Safran du Gâtinais, Pithiviers, Imprimerie Lucien Gauthier.

Veiga, I., 1999. Savoirs locaux et organisation sociale de l'agriculture familiale amazonienne : la gestion durable des milieux en question. Thèse, Université Toulouse Le Mirail.

Viard, N., 2001. Pratiques et connaissances autour de la culture du safran dans le cadre de la relance de la production de safran dans le Lot. Mémoire de fin d'études, INA P-G, Paris.

Walker, D.H., Thorne, P.J., Sinclair, F.L., Thapa, B., Wood, C.D., Subba, D.B., 1999. A systems approach to comparing indigenous and scientific knowledge: consistency and discriminatory power of indigenous and laboratory assessment of the nutritive value of tree fodder, Agricultural systems, 62, 2, 87-103.

Warren-Wilson J., 1972. Control of crop processes, in Rees, A.R., Cockshull, K.E., Hand, D.W., Hurd, R.G. (Eds), Crop Processes in Controlled Environment, London, Academic press, 7-30.

Zurayk, R., El-Awar, F., Hamadeh, S., Talhouk, S., Sayegh, C., Chehab, A.G., Al Shab, K., 2001. Using indigenous knowledge in land use investigations: a participatory study in a semi-arid mountainous region of Lebanon, Agriculture, Ecosystems and Environment, 86, 3, 247-262.

Reçu le 24 février 2004. Accepté le 4 novembre 2004.

To access this journal online: www.edpsciences.org 